\title{
Cost and Hospital Resource Utilization of Staphylococcus aureus Infection Post Elective Posterior Instrumented Spinal Fusion Surgeries in U.S. Hospitals: A Retrospective Cohort Study
}

\author{
Ning A. Rosenthal, MD, MPH, PhD, ${ }^{*}$ Kirstin $\mathrm{H}$. Heinrich, $\mathrm{MPH},{ }^{\dagger}$ Jessica Chung, $\mathrm{PhD}, \mathrm{MPH},{ }^{*}$ \\ and Holly $\mathrm{Yu}, \mathrm{MSPH}^{\dagger}$
}

\begin{abstract}
Study Design. A retrospective cohort study.
Objective. The aim of this study was to assess hospital resource utilization and costs associated with Staphylococcus aureus infection within 180 days post elective posterior instrumented spinal fusion surgeries (index surgery) between 2010 and 2015.
\end{abstract}

Summary of Background Data. Surgical site infections (SSIs) and blood stream infections (BSIs) post spinal fusion surgeries are associated with worse clinical outcomes and increased costs. Economic data specific to the most common pathogen of infections post spinal fusion surgeries, $S$. aureus, are limited.

Methods. We analyzed hospital discharge and microbiology data from 129 U.S. hospitals in Premier Healthcare Database. Selection criteria included age $\geq 18$ years; had a primary/ secondary ICD-9-CM procedure code for index surgery; and had microbiology data during study period. Outcomes included total hospitalization cost, length of stay, and risk of all-cause readmission. Infection status was classified as culture-confirmed invasive (i.e., BSIs, deep or organ/space SSIs), any, and no S. aureus infection. Multivariable regression analyses were used to compare outcome variables between infection groups controlling for known confounders.

From the *Premier Applied Sciences, Premier Healthcare Solutions Inc., Charlotte, NC; and 'Pfizer, Inc., Collegeville, PA.

Acknowledgment date: April 6, 2018. First revision date: August 2, 2018. Second revision date: September 9, 2018. Acceptance date: September 14, 2018.

The manuscript submitted does not contain information about medical device(s)/drug(s).

Pfizer Inc. funds were received in support of this work.

Relevant financial activities outside the submitted work: consultancy, employment.

This is an open access article distributed under the terms of the Creative Commons Attribution-Non Commercial-No Derivatives License 4.0 (CCBYNC-ND), where it is permissible to download and share the work provided it is properly cited. The work cannot be changed in any way or used commercially without permission from the journal.

Address correspondence and reprint requests to Ning A. Rosenthal, MD, $\mathrm{MPH}, \mathrm{PhD}$, Principal Research Scientist, Premier Healthcare Solutions Inc.,

13034 Ballantyne Corporate Place, Charlotte, NC 28277;

E-mail: ning_rosenthal@premierinc.com

DOI: 10.1097/BRS.0000000000002898

Spine
Results. Two hundred ninety-four patients had any $S$. aureus infection (151 had invasive infection) and 12,918 had no infection. Compared with no infection group, invasive and any infection groups had higher total hospitalization cost (adjusted mean in 2015 U.S. dollars: \$88,353 and \$64,356 vs. \$47,366, $P<0.001$ ), longer length of stay (adjusted mean: 20.98 and 13.15 vs. 6.77 days, $P<0.001)$, and higher risk of all-cause readmission [adjusted risk ratio: 2.15 (95\% confidence interval: 2.06-2.25) for invasive and 1.70 (95\% confidence interval: $1.61-1.80)$ for any infection groups].

Conclusion. $S$. aureus infections post elective posterior instrumented spinal fusion surgeries are associated with significantly higher hospitalization cost, length of stay, and 180-day risk of readmission than those with no such infection, which presents substantial burden to hospitals and patients. Reducing such infections may cut costs and hospital resource utilization.

Key words: cost, hospital resource utilization, postsurgical infection, spinal fusion, Staphylococcus aureus.

Level of Evidence: 3

Spine 2019;44:637-646

pinal fusion surgeries are common procedures for treating a variety of spinal conditions, including degenerative spine disease, scoliosis, mechanical back pain, spinal stenosis, isthmic spondylolisthesis, cervical myelopathy, fractures, and tumors. ${ }^{1-3}$ With the advancement in fusion devices and the increase of aging population, the use of spinal fusion procedures have increased dramatically since 1990s in the United States (U.S.). ${ }^{3-5}$ During 1997 to 2003, the utilization of cervical, thoracolumbar, and lumbar fusions increased by $89 \%, 31 \%$, and $134 \%$, respectively. ${ }^{4}$ A study using the Nationwide Inpatient Sample estimated that between 1998 and 2008, 1,288,496 primary posterior lumbar fusions were performed. ${ }^{6}$ Among Medicare patients, the rate of complex spinal fusion procedures increased 15-fold between 2002 and 2007 resulting in increased life-threatening complications, higher readmission rate, and greater hospitalization costs. ${ }^{3}$ Despite the extensive efforts being made to reduce infections among 
patients undergoing spinal fusion surgeries, postsurgical infections including surgical site infections (SSIs) and blood stream infections (BSIs) still remain constant threats to these patients, especially to those receiving open posterior instrumented spinal fusion procedures., ${ }^{3,70}$ With increasing emphasis on quality of care, reducing readmission rates and related costs has become an important component of health care reform in the U.S. Through establishing the Hospital Readmissions Reduction Program, the Centers for Medicare \& Medicaid Services has been required to reduce payments to hospitals with excess readmissions since $2012 .^{11}$

The risk of SSIs among patients undergoing instrumented spinal fusion surgeries was estimated to be $3.8 \%$ (median, $4.2 \%$; range, $0.4-20 \%$ ) based on 39 cohorts with a total of 28,628 patients. ${ }^{12}$ SSIs following spinal fusion surgeries can be superficial wound infection or invasive infection occurring in deep wound or organ space. ${ }^{13}$ Among all the pathogens causing postsurgical infections, Staphylococcus aureus (S. aureus) accounts for nearly half of all cases. , $^{8,13,14}$

SSIs and BSIs post spinal fusion surgeries were reported to be associated with worse clinical outcomes and significant cost increase compared with those without such complications. ${ }^{15-18}$ However, prior studies had small sample size, were conducted in a single health care facility, or did not focus specifically on $S$. aureus, the most common pathogen. For example, Kuhns et $a l^{18}$ reported that deep wound infection among 22 patients undergoing dorsal cervical fusion was associated with an average of $\$ 12,619$ higher cost than those without such infections. To better understand hospital resource utilization and costs related to postsurgical $S$. aureus infections among patients undergoing elective posterior instrumented spinal fusion surgeries in the U.S., use of data from large hospital discharge database would be beneficial.

This study aimed to assess the cost and hospital resource utilization related to postsurgical $S$. aureus SSIs and BSIs during 180-day follow-up period post elective, posterior, instrumented spinal fusion surgeries between 2010 and 2015 using data from 129 U.S. hospitals that consistently submitted microbiology data to the Premier Healthcare Database (PHD).

\section{MATERIALS AND METHODS}

\section{Study Design, Data Source, and Participants}

A retrospective cohort study using de-identified PHD data was conducted. The PHD is a complete census of inpatient and hospital-based outpatient visits from over 700 hospitals across all 50 states and contains $20 \%$ of all hospital discharges in the U.S. since 2000. PHD data are extracted from standard hospital discharge files and include patients' demographics, disease status, and information on date-stamped billed services in patients' daily service records. Patients can be tracked across visits within facilities with a unique identifier. ${ }^{19,20}$ About $25 \%$ of participating hospitals voluntarily contribute microbiology data to the PHD.

PHD data are HIPAA compliant according to 45 CRF 46.101(b)(4) and 45 CRF 164.506(d)(2)(ii)(B).
Patients who met all of the following inclusion criteria and none of the exclusion criteria were included. Inclusion criteria included (1) aged $\geq 18$ years at time of index surgery; $(2)$ had a principal/secondary ICD-9-CM procedure code for posterior instrumented spinal fusion surgeries including 81.01, 81.03, $81.05,81.07,81.08,81.31,81.33,81.35,81.37$, or 81.38 during index hospitalization; (3) index hospitalization was elective; (4) was admitted between January 01, 2010, and June 30, 2015, to a qualifying hospital that submitted consistent microbiology data during the 12 months before and 6 months post index surgery. Exclusion criteria included (1) had a major surgery as defined by the National Healthcare Safety Network (NHSN) procedures done between index surgery and the positive culture; ${ }^{21}(2)$ had a positive $S$. aureus culture from a normally sterile site such as blood or cerebral spinal fluid (CSF) collected during the 12 months before or 2 days post index surgery; and (3) had the following infections present at admission of the index hospitalization: BSIs, SSI deep and organ/space infections, osteomyelitis, vertebral disc space infection, meningitis, and intra-abdominal infections defined by ICD-9-CM diagnosis codes.

\section{Study Variables}

Main exposure variable was infection status during the 180day follow-up period. Three infection status groups were identified: any $S$. aureus infection, invasive $S$. aureus infection, and no $S$. aureus infection. Any $S$. aureus infection referred to having nonsurveillance culture positive for $S$. aureus (including both invasive and superficial infections). Invasive $S$. aureus infection was defined as having cultureconfirmed BSI, deep SSI, or organ/space SSI. Specifically, BSI was defined as having a positive blood culture for $S$. aureus or having a positive nonblood nonsurveillance $S$. aureus culture and having one of the following ICD-9-CM codes: 038.11 (Methicillin-susceptible $S$. aureus septicemia), 038.12 (Methicillin-resistant S. aureus septicemia), 790.7 (bacteremia), and 038.10 (Staphylococcal septicemia, unspecified). Deep SSI was defined as (1) having a wound culture positive for $S$. aureus together with one of the following reoperation posterior spinal fusion specific ICD-9-CM procedure codes: 03.02, 03.09, 03.4, 77.19, $77.49,77.69,78.69,80.09,80.39$, or one of the reoperation nonspecific ICD-9-CM procedure codes: 81.07, 81.62, $81.63,81.64,38.97,81.35,81.37,81.38,81.39,83.02$, $83.09,83.14,83.44,83.45,84.51,86.04,86.22$; or (2) having a deep wound culture positive for $S$. aureus. Organ/ Space SSI was defined as having a CSF culture positive for $S$. aureus or having a positive culture for $S$. aureus from a qualified specimen (e.g., body fluid culture, tissue culture) together with an ICD-9-CM diagnosis codes of 730.0x, 730.2x, 324.1, 324.9, 996.66, 996.67, 567.xx, and 320.3 during the same hospitalization. No $S$. aureus infection group included patients who did not have any culture-confirmed $S$. aureus infections. ICD code descriptions were listed in Appendix A, http://links.lww.com/BRS/B395.

Main outcomes included total and variable hospitalization costs, hospital length of stay, number and risk of readmission, 
16547

Unique patients meeting all inclusion criteria

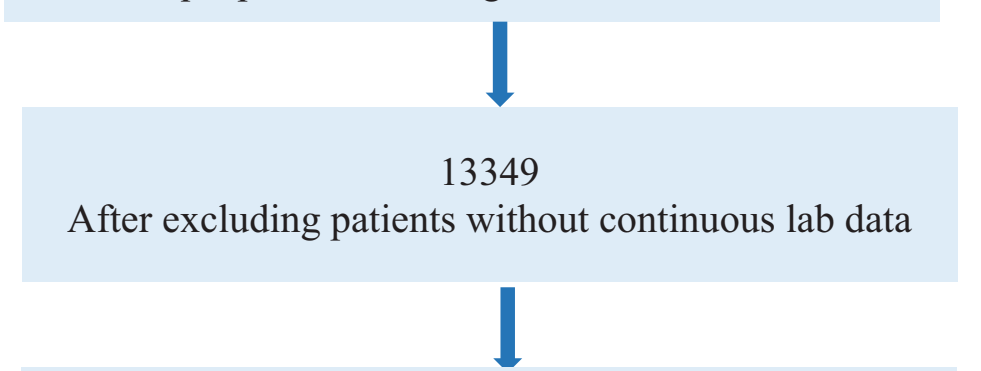

13212

After applying the three exclusion criteria

294

Any $S$. aureus infection

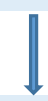

\section{1}

Invasive $S$. aureus infection

Figure 1. Staphylococcus aureus infection post spinal fusion surgery study patient selection flow chart.

and discharge status for the last hospital admission during follow-up. Variable cost was captured as directly reported by the hospitals, which typically includes cost that are deemed directly related to patient's clinical care (e.g., salaries for clinical staff, cost of supplies, and/or medications).

Patient and clinical characteristics that were assessed included sex, age, race/ethnicity, insurance type, level of fusion (single $v s$. multilevel), diabetic status, surgical history, year, type, and location of index surgery. Type of index surgery was categorized as fusion (Cervical: 81.01, 81.03; Thoracolumbar/Lumbar: 81.05, 81.07, 81.08), refusion (Cervical: 81.31, 81.33; Thoracolumbar/Lumbar: 81.35, 81.37 , or 81.38 ), and both fusion and refusion (having codes from both of the above categories). Fusion categories were mutually exclusive. Charlson Comorbidity Index (CCI) was assessed using ICD-9-CM diagnosis codes at index hospitalization using Deyo algorithm ${ }^{22}$ with Premier modifications. ${ }^{23}$ Hospital characteristics included hospital size, teaching status, population served (rural $v$ s. urban), and region.

\section{Statistical Methods}

Continuous data were expressed as mean, standard deviation, median, interquartile range, and range. Categorical data were expressed as counts and percentages of patients in each category. Chi-square tests were used for testing statistical differences between infection and no infection groups for categorical variables. T-test or Wilcoxon Rank Sum test was used for testing differences in continuous variables between comparison groups. Cost estimates were adjusted Spine to 2015 U.S. dollars based on Consumer Price Index for urban consumers for hospital and related services. ${ }^{24}$

Crude means were reported for cost variables by infection status. Generalized linear regression modeling was used to compare differences in cost between infection and no infection groups adjusting for known confounders, including age, race, fusion type, fusion level, CCI, hospital size, teaching status, and hospital region. Each model was bootstrapped using 1000 replicates with replacement to account for skewness in the data. For each group, mean, $2.5 \%$ and $97.5 \%$ estimates were used to determine the average costs and $95 \%$ confidence intervals (95\% CIs).

Negative binomial regression modeling was used to compare total length of stay and number of readmissions and Poisson regression modeling with robust error variance analysis was used to compare risk of readmission adjusting for known confounders.

Analyses were performed using SAS/STAT software, Version 9.4 of the SAS system [Copyright (2016) SAS Institute Inc., Cary, NC). Statistical significance level was assessed at 0.05 alpha level.

\section{RESULTS}

\section{Patient and Hospital Characteristics}

A total of 13,212 patients were included in the final analysis: $294(2.22 \%)$ with any S. aureus infection, including 151 $(1.14 \%)$ with invasive $S$. aureus infection and 12,918 with no $S$. aureus infection (Figure 1). Most patient characteristics 


\begin{tabular}{|c|c|c|c|c|c|}
\hline Characteristics & $\begin{array}{l}\text { No S. aureus } \\
\text { Infection } \\
\text { (Comparison } \\
\text { Group) }\end{array}$ & $\begin{array}{l}\text { Invasive } \\
\text { S. aureus } \\
\text { Infection }\end{array}$ & $\begin{array}{l}P \text { (Invasive } v s \text {. } \\
\text { No Infection) }\end{array}$ & $\begin{array}{l}\text { Any } S \text {. aureus } \\
\text { Infection }\end{array}$ & $\begin{array}{l}P \text { (Any vs. No } \\
\text { Infection) }\end{array}$ \\
\hline Total number of unique patients & 12,918 & 151 & & 294 & \\
\hline \multicolumn{6}{|l|}{ Patient characteristics } \\
\hline Male sex $(\%)$ & 42.69 & 36.42 & 0.2993 & 42.86 & 0.9872 \\
\hline Mean age in yrs (Std. Dev.) & $60.97(13.51)$ & $60.61(13.81)$ & 0.7793 & $60.24(13.80)$ & 0.3697 \\
\hline Race, white (\%) & 81.43 & 82.12 & 0.8280 & 87.41 & 0.0089 \\
\hline Multiple level of fusion (\%) & 69.25 & 81.46 & 0.0012 & 73.13 & 0.1540 \\
\hline \multicolumn{6}{|l|}{ Type of index surgery $(\%)$} \\
\hline Fusion & 92.26 & 91.39 & 0.0972 & 93.54 & 0.1129 \\
\hline Refusion & 5.05 & 3.31 & & 2.72 & \\
\hline Both fusion and refusion & 2.69 & 5.30 & & 3.74 & \\
\hline \multicolumn{6}{|l|}{ Year of index surgery $(\%)$} \\
\hline 2010 & 24.34 & 30.46 & 0.4521 & 23.13 & 0.1726 \\
\hline 2011 & 20.04 & 21.85 & & 26.19 & \\
\hline 2012 & 20.88 & 15.89 & & 20.07 & \\
\hline 2013 & 17.77 & 16.56 & & 17.01 & \\
\hline 2014 & 11.74 & 10.60 & & 9.18 & \\
\hline 2015 & 5.23 & 4.64 & & 4.42 & \\
\hline \multicolumn{6}{|l|}{ Location of index surgery (\%) } \\
\hline Thoracolumbar/lumbar & 87.20 & 88.74 & 0.3118 & 88.44 & 0.8023 \\
\hline Cervical & 11.81 & 9.27 & & 10.54 & \\
\hline $\begin{array}{l}\text { Both cervical and thoracolumbar/ } \\
\text { lumbar }\end{array}$ & 0.99 & 1.99 & & 1.02 & \\
\hline $\begin{array}{l}\text { Had surgery during the } 3 \text { months } \\
\text { before index surgery }(\%)\end{array}$ & 2.24 & 3.31 & 0.3967 & 1.70 & 0.5323 \\
\hline Prophylactic antibiotic use (\%) & 98.07 & 96.69 & 0.2219 & 98.30 & 0.7794 \\
\hline \multicolumn{6}{|l|}{ Hospital characteristics } \\
\hline \multicolumn{6}{|l|}{ Hospital size $(\%)$} \\
\hline $1-299$ beds & 32.47 & 23.84 & $<0.001$ & 43.88 & $<0.001$ \\
\hline $300-499$ beds & 44.96 & 39.07 & & 35.03 & \\
\hline $500+$ beds & 22.57 & 37.09 & & 21.09 & \\
\hline Teaching hospital (\%) & 42.88 & 50.33 & 0.0659 & 34.01 & 0.0024 \\
\hline Population served, rural (\%) & 4.47 & 4.64 & 0.9203 & 2.72 & 0.1502 \\
\hline \multicolumn{6}{|l|}{ Region of hospital (\%) } \\
\hline Midwest & 15.81 & 21.85 & 0.0206 & 12.93 & $<0.001$ \\
\hline Northeast & 20.84 & 21.85 & & 15.65 & \\
\hline South & 37.35 & 40.40 & & 56.46 & \\
\hline West & 26.00 & 15.89 & & 14.97 & \\
\hline
\end{tabular}

were comparable between infection and no infection groups (Table 1). Compared with no $S$. aureus infection group, a higher percentage of patients in invasive group had multilevel fusion $(81.46 \%$ vs. $69.25 \%, P=0.0012)$. Any infection group had similar distribution in all but race variables with a higher percentage of white patients than in no $S$. aureus infection group. There was significant difference in geographical location and hospital size between invasive and no S. aureus infection groups (Table 1).

\section{Baseline Charlson Comorbidities}

Only prevalence of diabetes without chronic complications varied significantly between groups (Table 2). A higher percentage of patients in invasive $(32.45 \%, P<0.001)$ and any $(26.53 \%, P=0.0074)$ infection groups had diabetes without chronic complications during the index hospitalization than no $S$. aureus infection group (20.39\%). Patients in invasive infection group had higher mean CCI score than those in no $S$. aureus infection group (1.09 vs. 0.83, $P=0.0006)$.

\section{Unadjusted Analysis Results}

All-cause 180-day readmission risk was $96.03 \%$ in invasive infection group, $54.76 \%$ in any infection group, and $19.18 \%$ in no $S$. aureus infection group $(P<0.001)$ (Table 3). Total length of hospital stay during index 


\begin{tabular}{|c|c|c|c|c|c|}
\hline Variables & $\begin{array}{l}\text { No } S \text {. aureus } \\
\text { Infection (Com- } \\
\text { parison Group) }\end{array}$ & $\begin{array}{l}\text { Invasive } \\
\text { S. aureus } \\
\text { Infection }\end{array}$ & $\begin{array}{l}P \text { (Invasive vs. } \\
\text { No Infection) }\end{array}$ & $\begin{array}{l}\text { Any } S . \text { aureus } \\
\text { Infection }\end{array}$ & $\begin{array}{c}P \text { (Any vs. } \\
\text { No Infection) }\end{array}$ \\
\hline Total number of unique patients & 12,918 & 151 & & 294 & \\
\hline \multicolumn{6}{|l|}{ Type of comorbidities (\%) } \\
\hline Myocardial infarction & 4.34 & 5.96 & 0.3306 & 4.42 & 0.9424 \\
\hline Congestive heart failure & 3.51 & 5.96 & 0.1045 & 3.40 & 0.9226 \\
\hline Peripheral vascular & 2.80 & 4.64 & 0.2059 & 3.40 & 0.5392 \\
\hline Cerebrovascular disease & 2.14 & 1.99 & 1.0000 & 2.04 & 0.9035 \\
\hline Dementia & 0.63 & 0.66 & 0.6200 & 1.36 & 0.1249 \\
\hline Chronic pulmonary disease & 20.09 & 23.84 & 0.2529 & 18.71 & 0.5588 \\
\hline Rheumatic disease & 4.30 & 5.30 & 0.5501 & 4.76 & 0.7024 \\
\hline Peptic ulcer disease & 0.63 & 0.66 & 0.6155 & 0.68 & 0.7081 \\
\hline Mild liver disease & 0.35 & 0.66 & 0.4146 & 0.34 & 1.0000 \\
\hline $\begin{array}{l}\text { Diabetes without chronic } \\
\text { complication }\end{array}$ & 20.39 & 32.45 & $<0.001$ & 26.53 & 0.0099 \\
\hline $\begin{array}{l}\text { Diabetes with chronic } \\
\text { complication }\end{array}$ & 2.93 & 1.32 & 0.3315 & 1.02 & 0.0529 \\
\hline Hemiplegia or paraplegia & 0.55 & 1.99 & 0.0541 & 1.02 & 0.2274 \\
\hline Renal disease & 5.41 & 5.30 & 0.9513 & 4.08 & 0.3179 \\
\hline Moderate or severe liver disease & 0.11 & 0.66 & 0.1601 & 0.34 & 0.2866 \\
\hline Any malignancy* & 1.18 & 1.99 & 0.2662 & 1.02 & 1.0000 \\
\hline Metastatic solid tumor ${ }^{\dagger}$ & 0.53 & 0.66 & 0.5525 & 0.34 & 1.0000 \\
\hline AIDS/HIV & 0.07 & 0.00 & 0.7456 & 0.00 & 0.6507 \\
\hline Mean CCI Score (Std. Dev.) & $0.83(1.23)$ & $1.09(1.28)$ & 0.0006 & $0.83(1.11)$ & 0.4500 \\
\hline \multicolumn{6}{|c|}{$\begin{array}{l}\text { No S. aureus infection group is the comparison group for both invasive and any S. aureus infection groups. } \\
\text { S. aureus indicates Staphylococcus aureus. } \\
\text { "Including lymphoma and leukemia, not include malignant neoplasm of skin. }\end{array}$} \\
\hline
\end{tabular}

hospitalization and follow-up in invasive infection group was over three times that of no $S$. aureus infection group. Consistent with readmission risk and hospital length of stay observed, total and variable hospitalization costs were the highest in invasive group, followed by any and no $S$. aureus infection groups. Compared with no $S$. aureus infection group, a higher percentage of patients in invasive group were discharged to nursing homes, long-term care facilities, hospice, or other acute care facilities $(49 \%$ vs. $32 \%)$ at their last hospital admission during follow-up (Table 3).

\section{Adjusted Analysis Results}

After adjusting for confounders and using bootstrapping method, the mean number of 180-day all-cause readmissions was highest among invasive infection group and lowest among no $S$. aureus infection group (adjusted mean: $1.65 \mathrm{vs}$. $0.25, P<0.001$ ) (Table 4). Mean length of hospital stay during index hospitalization and 180-day follow-up period in invasive group was three times of that in no $S$. aureus infection group (adjusted mean: 20.98 vs. 6.77 days, $P$ $<0.001)$. The adjusted mean length of stay in the any infection group was two times of that in no $S$. aureus infection group (adjusted mean: 13.15 vs. 6.77 days, $P$ $<0.001)$. The adjusted mean of total hospitalization cost remained highest in invasive infection group (Mean:
$\$ 88,353,95 \%$ CI: 78,907-100,893), followed by any infection group (Mean: \$64,356, 95\% CI: 58,106-70,893), and lowest in no $S$. aureus infection group (Mean: $\$ 47,366,95 \%$ CI: 46,840-47,889) (Figure 2). The adjusted total variable hospitalization cost followed similar patterns and was highest among patients with invasive infection (Mean: \$50,966, 95\% CI: 45,802-57,975), followed by any infection group (Mean: \$39,820, 95\% CI: 36,199-43,673), and lowest in no $S$. aureus infection group (Mean: \$30,243, 95\% CI: 29,918-30,592).

Compared with patients under 50 years, patients $\geq 75$ years were twice as likely to get readmitted. Nonwhite patients and those with multilevel fusion, having both fusion and refusion surgeries during index hospitalization, having higher CCI, from medium size hospitals or hospitals in Midwest were more likely to get readmitted than their peers (Table 5). After controlling confounders, the relative risk of 180-day all-cause readmission in invasive and any infection group was 2.15 times (95\% CI: 2.06-2.25) and 1.70 times (95\% CI: $1.61-1.80)$ that of no $S$. aureus infection group, respectively.

\section{DISCUSSION}

As one of the first studies using large national hospital discharge data to assess hospital resource utilization and cost related to culture-confirmed $S$. aureus infections post 


\begin{tabular}{|c|c|c|c|c|c|}
\hline Outcomes & $\begin{array}{l}\text { No } S \text {. aureus } \\
\text { Infection } \\
\text { (Comparison } \\
\text { Group) }\end{array}$ & $\begin{array}{l}\text { Invasive } \\
\text { S. aureus } \\
\text { Infection }\end{array}$ & $\begin{array}{c}P \text { (Invasive } \\
v s . \\
\text { No Infection) }\end{array}$ & $\begin{array}{c}\text { Any } \\
\text { S. aureus* } \\
\text { Infection }\end{array}$ & $\begin{array}{c}P \text { (Any vs. } \\
\text { No Infection) }\end{array}$ \\
\hline Total number of unique patients & 12,918 & 151 & & 294 & \\
\hline Mean number of all-cause readmission (Std. Dev.) & $0.25(0.59)$ & $1.65(0.99)$ & $<0.001$ & $0.90(1.07)$ & $<0.001$ \\
\hline All-cause readmission rate $(\%)$ & 19.18 & 96.03 & $<0.001$ & 54.76 & $<0.001$ \\
\hline \multicolumn{6}{|l|}{ Total length of stay, days } \\
\hline Mean & 6.24 & 21.26 & $<0.001$ & 12.60 & $<0.001$ \\
\hline STD & 6.74 & 18.79 & & 16.24 & \\
\hline Median & 4.00 & 17.00 & & 6.00 & \\
\hline IQR & 3,7 & 9,28 & & 3,17 & \\
\hline Range & 1,189 & 4,121 & & 1,121 & \\
\hline \multicolumn{6}{|l|}{ Total hospitalization cost (in 2015 U.S. dollars) } \\
\hline Mean & $\$ 48,968$ & $\$ 98,382$ & $<0.001$ & $\$ 68,460$ & $<0.001$ \\
\hline STD & $\$ 33,674$ & $\$ 80,526$ & & $\$ 67,982$ & \\
\hline Median & $\$ 40,273$ & $\$ 79,569$ & & $\$ 49,516$ & \\
\hline IQR & $\begin{array}{l}\$ 27,747, \\
\$ 59,812\end{array}$ & $\begin{array}{l}\$ 53,122 \\
\$ 113,779 \\
\end{array}$ & & $\begin{array}{l}\$ 28,378, \\
\$ 83,397\end{array}$ & \\
\hline Range & $\$ 444,026$ & $\$ 586,069$ & & $\$ 591,722$ & \\
\hline \multicolumn{6}{|c|}{ Total variable hospitalization cost (in 2015 U.S. dollars) } \\
\hline Mean & $\$ 31,351$ & $\$ 57,144$ & $<0.001$ & $\$ 42,387$ & $<0.001$ \\
\hline STD & $\$ 22,578$ & $\$ 45,848$ & & $\$ 38,289$ & \\
\hline Median & $\$ 25,595$ & $\$ 43,983$ & & $\$ 31,580$ & \\
\hline IQR & $\begin{array}{l}\$ 17,225 \\
\$ 38,584\end{array}$ & $\begin{array}{l}\$ 31,559, \\
\$ 67,127\end{array}$ & & $\begin{array}{l}\$ 20,445 \\
\$ 51,565\end{array}$ & \\
\hline Range & $\$ 364,867$ & $\$ 388,928$ & & $\$ 389,854$ & \\
\hline \multicolumn{6}{|l|}{ Discharge status for latest inpatient admission (\%) } \\
\hline Home/Home health & 68.00 & 50.99 & $<0.001$ & 66.67 & 0.1419 \\
\hline Skilled nursing, hospice, rehabilitation centers & 31.34 & 47.02 & & 32.31 & \\
\hline Transfer to another acute care hospital & 0.27 & 1.99 & & 1.02 & \\
\hline Expired & 0.25 & 0.00 & & 0.00 & \\
\hline Other/unknown & 0.14 & 0.00 & & 0.00 & \\
\hline
\end{tabular}

elective posterior instrumented spinal fusion surgeries, our study demonstrated that nearly all patients $(96.03 \%)$ with invasive $S$. aureus infection had at least one readmission compared with $19.18 \%$ among no $S$. aureus infection group. After adjusting for confounders, invasive $S$. aureus infection was associated with a $115 \%$ increased risk of all-cause readmission during the 180-day follow-up period compared with no $S$. aureus infection group. Patients with invasive $S$. aureus infection usually need aggressive surgical debridement and prolonged antibiotic treatment, which often requires readmission. ${ }^{25}$ Using data from a single hospital, Schairer et $a l^{26}$ estimated that the reoperation rate for instrumented spinal fusion patients was $89.2 \%$, which is consistent with the high readmission rate reported by our study.

When comparing hospital resource utilization between infection groups, our findings indicated that invasive and any $S$. aureus infection groups had an average of 14.21 and 6.38 more days of hospital stay during the index hospitalization and follow-up than no $S$. aureus infection group after adjusting for confounders. Pooled results from the study by Patel et $a l^{12}$ also indicated that patients with any SSI had longer length of stay (range, 7.1-19.3 days) than those without SSI (4.0-9.3 days).

Higher readmission risk and longer total hospital length of stay in invasive and any postsurgical $S$. aureus infection groups resulted in an average of $\$ 40,987$ and $\$ 16,900$ more total hospitalization cost compared with no $S$. aureus infection group over the 180-day follow-up period, respectively. The infection groups also had higher total variable hospitalization cost than the no infection group, which indicated that patients with postsurgical $S$. aureus infections consume more clinical resources in the hospital than those without such infections. Although the absolute amount of extra cost estimated by our study differs from estimates of previous studies due to different cost variable definitions and study populations, the general association between postsurgical 


\begin{tabular}{|c|c|c|c|}
\hline Hospitalization Cost & Invasive $S$. aureus Infection & Any $S$. aureus Infection & No $S$. aureus Infection \\
\hline Total number of unique patients & 151 & 294 & 12,918 \\
\hline \multicolumn{4}{|c|}{ Total number of 180 -day readmissions } \\
\hline Adjusted mean & 1.65 & 0.94 & 0.25 \\
\hline $95 \% \mathrm{Cl}$ & $1.33-2.05$ & $0.78-1.14$ & $0.22-0.27$ \\
\hline \multicolumn{4}{|l|}{ Total length of stay, days } \\
\hline Adjusted mean & 20.98 & 13.15 & 6.77 \\
\hline $95 \% \mathrm{Cl}$ & $18.80-23.42$ & $12.07-14.33$ & $6.54-7.01$ \\
\hline \multicolumn{4}{|l|}{ Total hospitalization cost } \\
\hline Mean & $\$ 88,353$ & $\$ 64,356$ & $\$ 47,366$ \\
\hline $95 \% \mathrm{Cl}$ & $\$ 78,907-\$ 100,893$ & $\$ 58,106-\$ 70,893$ & $\$ 46,840-\$ 47,889$ \\
\hline \multicolumn{4}{|l|}{ Total variable hospitalization cost } \\
\hline Mean & $\$ 50,966$ & $\$ 39,820$ & $\$ 30,243$ \\
\hline $95 \% \mathrm{Cl}$ & $\$ 45,802-\$ 57,975$ & $\$ 36,199-\$ 43,673$ & $\$ 29,918-\$ 30,592$ \\
\hline \multicolumn{4}{|c|}{$\begin{array}{l}\text { (1) Bootstrapping was used in the modeling. Confounding variables adjusted in the models included age, race, fusion type, fusion level, Charlson Comorbidit } \\
\text { Index, hospital size, teaching status, and hospital region. (2) Estimates for No S. aureus infection group were from modeling comparisons between invasive } \\
\text { S. aureus and No S. aureus group. No S. aureus group is the reference group for each set of comparisons. (3) All cost calculation was adjusted to } 2015 \text { US } \\
\text { dollars based on Consumer Price Index for all urban consumers for hospital and related services. } \\
\text { Cl indicates confidence interval; S. aureus, Staphylococcus aureus. }\end{array}$} \\
\hline
\end{tabular}
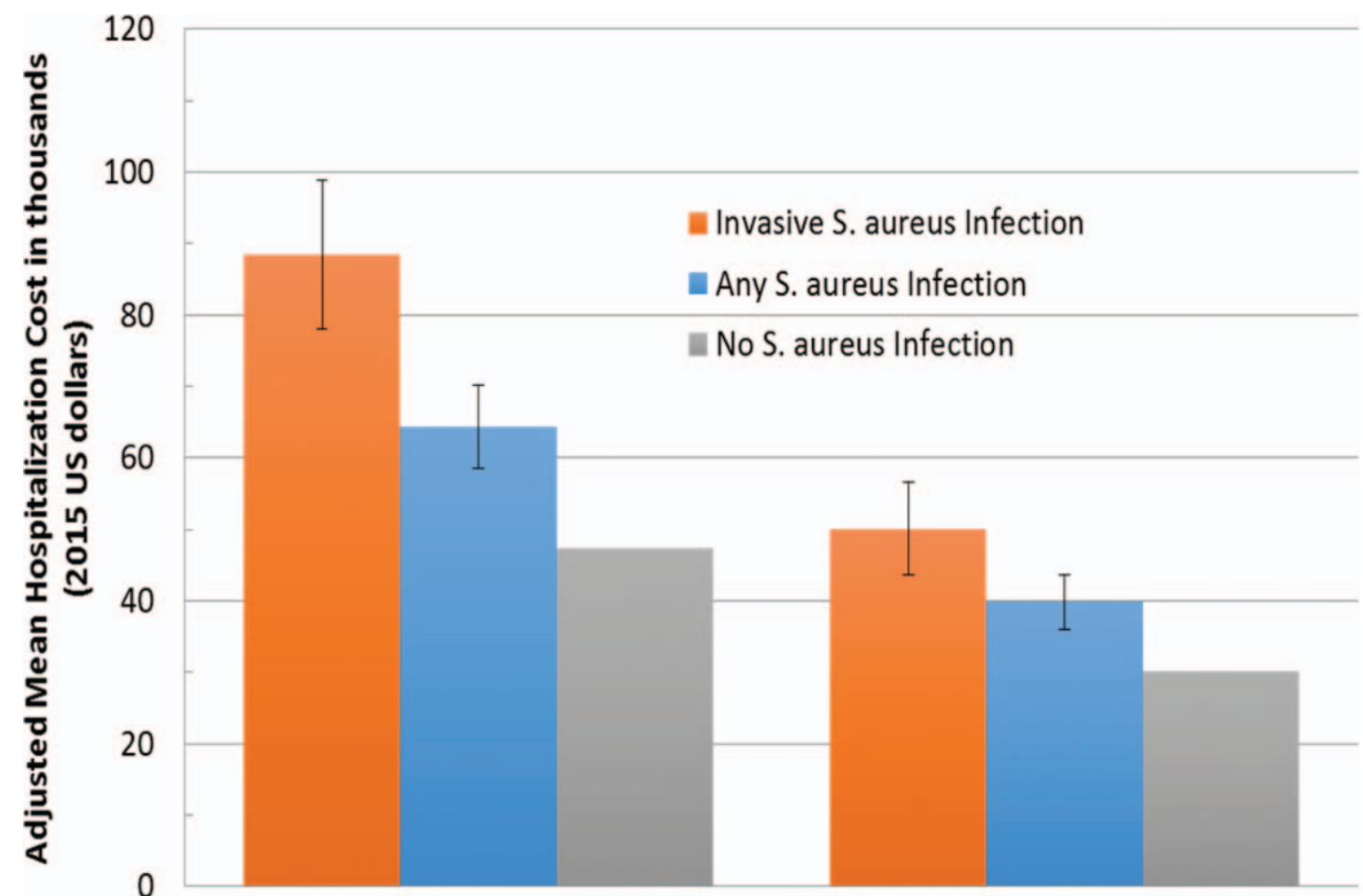

Total Costs

Variable Costs

\section{Cost Type}

Figure 2. Adjusted mean hospitalization cost and 95\% confidence interval by infection status. Solid black bar represents Invasive $S$. aureus Infection group, grey bar with line pattern represents Any Invasive S. aureus Infection group, and white bar with dot pattern represents No S. aureus Infection group. Note: The 95\% confidence intervals for the No $S$. aureus infection group were too narrow to show up in the chart but they did not overlap with intervals of the other two infection groups for either total or variable cost. 


\begin{tabular}{|c|c|c|c|c|}
\hline \multirow[b]{2}{*}{ Variable } & \multicolumn{2}{|c|}{$\begin{array}{l}\text { Model 1: Invasive vs. } \\
\text { No S. aureus Infection }\end{array}$} & \multicolumn{2}{|c|}{$\begin{array}{l}\text { Model 2: Any vs. } \\
\text { No } S \text {. aureus Infection }\end{array}$} \\
\hline & Risk Ratio & $95 \% \mathrm{Cl}$ & Risk Ratio & $95 \% \mathrm{Cl}$ \\
\hline Invasive $S$. aureus infection & 2.15 & $2.06-2.25$ & $\mathrm{~N} / \mathrm{A}$ & $\mathrm{N} / \mathrm{A}$ \\
\hline Overall S. aureus infection & $\mathrm{N} / \mathrm{A}$ & $\mathrm{N} / \mathrm{A}$ & 1.70 & $1.61-1.80$ \\
\hline \multicolumn{5}{|l|}{ Age in yrs $(\%)$} \\
\hline $18-49$ & Reference & Reference & Reference & Reference \\
\hline $50-64$ & 1.24 & $1.10-1.39$ & 1.22 & $1.09-1.37$ \\
\hline $65-74$ & 1.72 & $1.54-1.93$ & 1.69 & $1.51-1.89$ \\
\hline $75+$ & 2.06 & $1.82-2.32$ & 2.04 & $1.81-2.30$ \\
\hline \multicolumn{5}{|l|}{ Race (\%) } \\
\hline White & Reference & Reference & Reference & Reference \\
\hline Nonwhite & 1.09 & $1.05-1.14$ & 1.10 & $1.05-1.14$ \\
\hline \multicolumn{5}{|l|}{ Level of fusion (\%) } \\
\hline Single level & Reference & Reference & Reference & Reference \\
\hline Multiple level & 1.17 & $1.13-1.22$ & 1.18 & $1.13-1.23$ \\
\hline \multicolumn{5}{|l|}{ Type of index surgery $(\%)$} \\
\hline Fusion & Reference & Reference & Reference & Reference \\
\hline Refusion & 1.11 & $0.95-1.29$ & 1.11 & $0.95-1.30$ \\
\hline Both fusion and refusion & 1.28 & $1.08-1.52$ & 1.29 & $1.09-1.53$ \\
\hline Charlson Comorbidity Index & 1.13 & $1.11-1.16$ & 1.14 & $1.12-1.16$ \\
\hline \multicolumn{5}{|l|}{ Hospital size $(\%)$} \\
\hline $1-299$ beds & 0.80 & $0.75-0.85$ & 0.78 & $0.73-0.82$ \\
\hline $300-499$ beds & 1.10 & $1.05-1.16$ & 1.11 & $1.06-1.17$ \\
\hline $500+$ beds & Reference & Reference & Reference & Reference \\
\hline \multicolumn{5}{|l|}{ Teaching status (\%) } \\
\hline Nonteaching hospital & Reference & Reference & Reference & Reference \\
\hline Teaching hospital & 0.82 & $0.78-0.86$ & 0.83 & $0.79-0.87$ \\
\hline \multicolumn{5}{|l|}{ Region of hospital (\%) } \\
\hline Midwest & 1.14 & $1.07-1.22$ & 1.15 & $1.08-1.23$ \\
\hline Northeast & 1.00 & $0.92-1.09$ & 1.01 & $0.93-1.11$ \\
\hline South & 1.03 & $0.97-1.08$ & 1.00 & $0.95-1.06$ \\
\hline West & Reference & Reference & Reference & Reference \\
\hline
\end{tabular}

infection and higher health care cost remains consistent across studies. ${ }^{18,27}$ Because this is a study using existing hospital discharge data, we were only able to assess the hospital burdens of $S$. aureus infections post target spinal fusion surgeries. The overall burden to the healthcare system and to the society would be even more substantial.

Compared with studies published before, this study has multiple strengths. First, we used data collected from 129 hospitals nationwide, which is more representative than studies conducted in one single institution. The large sample size provided sufficient power to test differences in key outcomes between comparison groups. Second, all cases were culture-confirmed, which is more accurate than cases identified by ICD diagnosis codes only. Third, we included not only invasive SSI but also BSI and noninvasive infections in the analysis, which provided a broad spectrum of $S$. aureus infections for analysis while further segmenting the infections by invasiveness (invasive and any $S$. aureus infection). Finally, on the basis of hospital reporting, variable hospitalization costs could be assessed, which may reflect attributable clinical resource utilization more accurately than total hospitalization cost. ${ }^{28}$

This study also has limitations. First, although we have high-quality cost and clinical data, we miss some clinical details that may help differentiate superficial from invasive $S$. aureus infections. This limited our ability to assess the impact of superficial infections separately. Second, because PHD only captures readmissions or outpatient visits to the same hospitals where the index surgeries occurred, the number of $S$. aureus infections, number of readmissions, and costs may be underestimated. This limitation is expected to be nondifferential across infection groups. 
Third, we only focused on $S$. aureus infections among patients with posterior instrumented spinal fusion surgeries in this study. More research is needed to study the impact of infections due to other pathogens and among patients with other types of spinal fusion surgeries.

In conclusion, our findings indicate that among patients undergoing elective, posterior, instrumented spinal fusion surgeries, $S$. aureus infections, especially invasive infections, are associated with significantly higher hospitalization cost, risk and number of all-cause readmissions, and total length of hospital stay during the 180-day follow-up period postindex surgery compared with patients experiencing no $S$. aureus infection. Reducing postsurgical $S$. aureus infection risk among these patients may reduce risk of readmission and economic burden. Although the effectiveness of many preoperative, intraoperative, and postoperative infection control measures on reducing SSIs have been studied, few have been proven effective with strong clinical evidence support based on guidelines from the World Health Organization and the Association for Professionals in Infection Control and Epidemiology. ${ }^{29,30}$ The review by Agarwal et al on implant contamination and septic methods concluded that more research is needed to explore effective methods to prevent postsurgical infections among patients undergoing elective, posterior, instrumented spinal fusion surgeries. ${ }^{31}$

\section{Key Points}

- Staphylococcus aureus infections post elective posterior instrumented spinal fusion surgeries, especially invasive infections, are associated with significantly higher hospitalization cost, length of stay, and 180-day risk of readmission compared with those with no such infection.

- The overall 180-day risk of any Staphylococcus aureus infection was estimated to be $2.22 \%$ and of invasive Staphylococcus aureus infection was estimated to be $1.14 \%$.

- The substantial economic and clinical burden associated with postsurgical Staphylococcus aureus infections calls for more effective infection control measures to reduce such infections.

\section{Acknowledgment}

The authors would like to acknowledge Drs. Beth Begier and Alejandra Gurtman for their scientific input.

Supplemental digital content is available for this article. Direct URL citations appearing in the printed text are provided in the HTML and PDF version of this article on the journal's Web site (www.spinejournal.com).

\section{References}

1. Chaichana KL, Bydon M, Santiago-Dieppa DR, et al. Risk of infection following posterior instrumented lumbar fusion for degenerative spine disease in 817 consecutive cases. J Neurosurg Spine 2014;20:45-52.
2. Choy W, Lam SK, Smith ZA, Dahdaleh NS. Predictors of 30-Day Hospital Readmission After Posterior Cervical Fusion in 3401 Patients. Spine 2018;43:356-63.

3. Deyo RA, Mirza SK, Martin BI, et al. Trends, major medical complications, and charges associated with surgery for lumbar spinal stenosis in older adults. JAMA 2010;303:1259-65.

4. Cowan JA Jr, Dimick JB, Wainess R, et al. Changes in the utilization of spinal fusion in the United States. Neurosurgery 2006;59:15-20; discussion 15-20.

5. Deyo RA, Gray DT, Kreuter W, et al. United States trends in lumbar fusion surgery for degenerative conditions. Spine (Phila Pa 1976) 2005;30:1441-5; discussion 1446-1447.

6. Pumberger M, Chiu YL, Ma Y, et al. Perioperative mortality after lumbar spinal fusion surgery: an analysis of epidemiology and risk factors. Eur Spine J 2012;21:1633-9.

7. Gerometta A, Rodriguez Olaverri JC, Bitan F. Infections in spinal instrumentation. Int Orthop 2012;36:457-64.

8. Mackenzie WG, Matsumoto H, Williams BA, et al. Surgical site infection following spinal instrumentation for scoliosis: a multicenter analysis of rates, risk factors, and pathogens. J Bone Joint Surg Am 2013;95:800-6; s801-802.

9. Su AW, Habermann EB, Thomsen KM, Milbrandt TA, Nassr A, Larson AN. Risk factors for 30-day unplanned readmission and major perioperative complications after spine fusion surgery in adults: a review of the national surgical quality improvement program database. Spine 2016;41:1523-34.

10. Wang MC, Chan L, Maiman DJ, et al. Complications and mortality associated with cervical spine surgery for degenerative disease in the United States. Spine (Phila Pa 1976) 2007;32:342-7.

11. Centers for Medicare \& Medicaid Services. Readmissions Reduction Program. Available at: https://www.cms.gov/Medicare/Medicare-Fee-for-Service-Payment/AcuteInpatientPPS/ReadmissionsReduction-Program.html. Accessed May 23, 2017.

12. Patel H, Khoury H, Girgenti D, et al. Burden of surgical site infections associated with select spine operations and involvement of Staphylococcus aureus. Surg Infect (Larchmt) 2017;18:461-73.

13. Chahoud J, Kanafani Z, Kanj SS. Surgical site infections following spine surgery: eliminating the controversies in the diagnosis. Front Med (Lausanne) 2014;1:7.

14. Takizawa T, Tsutsumimoto T, Yui M, et al. Surgical site infections caused by methicillin-resistant Staphylococcus epidermidis following spinal instrumentation surgery. Spine (Phila Pa 1976) 2017; 42:525-30.

15. Calderone RR, Garland DE, Capen DA, et al. Cost of medical care for postoperative spinal infections. Orthop Clin North Am 1996; 27:171-82.

16. Chen $\mathrm{SH}$, Lee $\mathrm{CH}$, Huang $\mathrm{KC}$, et al. Postoperative wound infection after posterior spinal instrumentation: analysis of long-term treatment outcomes. Eur Spine J 2015;24:561-70.

17. Drazin D, Nuno M, Shweikeh F, et al. Outcomes and national trends for the surgical treatment of lumbar spine trauma. Biomed Res Int 2016;2016:3623875.

18. Kuhns BD, Lubelski D, Alvin MD, et al. Cost and quality of life outcome analysis of postoperative infections after subaxial dorsal cervical fusions. I Neurosurg Spine 2015;22:381-6.

19. Magee G, Zaloga GP, Turpin RS, et al. A retrospective, observational study of patient outcomes for critically ill patients receiving parenteral nutrition. Value Health 2014;17:328-33.

20. Pontes-Arruda A, Liu FX, Turpin RS, et al. Bloodstream infections in patients receiving manufactured parenteral nutrition with vs without lipids: is the use of lipids really deleterious?. JPEN J Parenter Enteral Nutr 2012;36:421-30.

21. Centers for Disease Control and Prevention National Healthcare Safety Network. Surgical Site Infection (SSI) Event. 2018. Available at: https://www.cdc.gov/nhsn/pdfs/pscmanual/9pscssicurrent.pdf. Accessed January 5, 2018.

22. Deyo RA, Cherkin DC, Ciol MA. Adapting a clinical comorbidity index for use with ICD-9-CM administrative databases. J Clin Epidemiol 1992;45:613-9.

23. Rosenthal NA, Cao Z, Chung J, et al. Updated Coding Algorithm for Assessing Charlson Comorbidity Index Using Large Hospital 
Administrative Data. Presented at the 2017 ISPOR Annual Conference; May 21, 2017; Boston, MA.

24. US Department of Labor, Bureau of Labor Statistics. Inpatient Hospital Services Consumer Price Index. Available at: http:// www.bls.gov/cpi/. Accessed March 4, 2017.

25. Maruo K, Berven SH. Outcome and treatment of postoperative spine surgical site infections: predictors of treatment success and failure. J Orthop Sci 2014;19:398-404.

26. Schairer WW, Carrer A, Deviren V, et al. Hospital readmission after spine fusion for adult spinal deformity. Spine (Phila Pa 1976) 2013;38:1681-9.

27. Parker SL, Shau DN, Mendenhall SK, et al. Factors influencing 2-year health care costs in patients undergoing revision lumbar fusion procedures. J Neurosurg Spine 2012;16: $323-328$.
28. Obeid T, Alshaikh H, Nejim B, et al. Fixed and variable cost of carotid endarterectomy and stenting in the United States: a comparative study. J Vasc Surg 2017;65:1398-1406.e1.

29. Association for Professionals in Infection Control and Epidemiology. Guide to the Elimination of Orthopedic Surgical Site Infections. 2010. Available at: https://apic.org/Resource_/EliminationGuideForm/34e03612-d1e6-4214-a76b-e532c6fc3898/File/APIC-OrthoGuide.pdf. Accessed June 28, 2018.

30. The World Health Organization. Global Guidelines for the Prevention of Surgical Site Infection. 2016. Available at: https://www. ncbi.nlm.nih.gov/books/NBK401129/. Accessed June 28, 2018.

31. Agarwal A, Schultz C, Goel VK, et al. Implant prophylaxis: the next best practice toward asepsis in spine surgery. Global Spine J 2018; Accessed online at: http://journals.sagepub.com/doi/abs/ $10.1177 / 2192568218762380 \#$ on June 28th, 2018. 